\title{
Uncoordinated Beamforming for Cognitive Networks
}

\author{
Simon Yiu, Chan-Byoung Chae, Kai Yang, and Doru Calin \\ Bell Labs, Alcatel-Lucent, USA \\ Email: \{simon.yiu, chan-byoung.chae, kai.yang, doru.calin\}@alcatel-lucent.com
}

\begin{abstract}
In this paper, we propose jointly-optimized beamforming algorithms for cognitive networks to maximize the achievable rates assuming single information stream in both primary and secondary links. The primary and cognitive users share the same spectrum and are equipped with multiple antennas. No coordination is required between the primary and cognitive users and the interference cancellation is done at the cognitive user. Specifically, the beamforming vectors of the cognitive link are designed to maximize the achievable rate under the condition that the interference both at the primary and cognitive receivers is completely nullified. The sum rate performance of the proposed algorithms is evaluated by Monte Carlo simulations.
\end{abstract}

\section{INTRODUCTION}

The opportunistic use of the wireless spectrum has been a hot research topic in the wireless communications community in recent years due to the intense competition for the use of spectrum at frequencies below $3 \mathrm{GHz}$ [1]. In particular, cognitive networks have received much attention [2]. A cognitive network consists of a number of traditional wireless service subscribers and the so-called cognitive users. The traditional wireless service subscribers have the legacy priority access to the spectrum and are usually called the primary users in the literature. On the other hand, cognitive users, which are also known as the secondary users, are allowed to access the spectrum only if communication does not create significant interference to the licensed primary users. This can be achieved in several ways as discussed in [3] and references therein. For example, the cognitive user can transmit concurrently with the primary users under an enforced spectral mask. Another strategy, commonly referred to as spectrum sensing, is to have the cognitive users monitor the spectrum and access it when an unused slot is detected.

Since the primary user network should not be required to change their infrastructure and topology for spectrum sharing with the secondary users, the secondary users should be able to detect the presence of primary users in the spectrum independently and efficiently. Spectrum sensing [4] is the fundamental problem that many researchers attempt to address in the literature [5], [6], [7]. The problem is essentially a detection where the goal is to find an optimal decision threshold. The design of the threshold creates an interesting trade-off between the probability of miss detection and the probability of false alarm. A low probability of miss detection can be achieved by using a low threshold at an expense of high probability of false alarm. Likewise, one can design a system with a low probability of false alarm by utilizing a high threshold at an expense of high probability of miss detection. A high probability of false alarm is not desirable because the cognitive user remains silent even when the spectrum is not being used by the primary user. On the other hand, the cognitive user constantly creates interference to the primary user in a system with a high probability of miss detection.

In this paper, we address the interference problem in cognitive networks from another perspective. There is no spectrum detection (sensing) involved and both primary and secondary users can transmit simultaneously through the same spectrum without interfering each others. In [8], the authors proposed opportunistic spectrum sharing algorithms by exploiting multi-antennas but they mostly focused on removing interference only from a secondary transmitter to primary receivers. Unlike prior work, in this paper, we propose uncoordinated beamforming techniques in cognitive networks to completely remove the cross interference between the primary and secondary users. The proposed algorithms also maximize the achievable rates of both links through uncoordinated beamforming. Beamforming is a well-studied subject in the signal processing literature. It can be used for either directed transmission or reception of energy in the presence of noise and interference [9].

In this paper, we consider a cognitive network that consists of a single primary and secondary user. Each user consists of a transmitter and a receiver. Both transmitters and receivers are equipped with multiple antennas and beamforming transmit/receive vectors. In particular, we claim the following contributions:

- We propose three designs for the beamforming vectors. The beamforming vectors are designed such that the interference caused by the cognitive transmitter to the primary receiver and the interference caused by the primary transmitter to the cognitive receiver is completely nullified while maximizing the rate of both the primary and secondary links.

- The proposed designs do not require knowledge of the cognitive communication link at the primary user. In fact, the secondary user is invisible to the primary user and the performance of the primary link is not affected by the secondary user at all. This is in contrary to prior work in the literature which requires coordination between the primary and secondary users [10], [11], [12], [13].

Organization: This paper is organized as follows. In Section II, we introduce the system model of the proposed transmission scheme and formulate the beamforming optimization problem. In Section III, we propose three beamforming solutions which cancel the interference both at the primary and secondary receiver while maximizing the spectral efficiency 
of both the primary and secondary links. Finally, we present some simulation and numerical results in Section IV, and draw some conclusions in Section V.

Notation: In this paper, bold upper case and lower case letters denote channel matrices ${ }^{1}$ and beamforming vectors, respectively. $[\cdot]^{*}, \mathbb{E}\{\cdot\}, \mathrm{j} \triangleq \sqrt{-1}, \mathbb{C}, \operatorname{Null}(\cdot), \lambda_{\max }(\cdot)$, and $\mathbf{1}_{N}$ denote Hermitian transposition, statistical expectation, the imaginary unit, the set of complex number, the null space of a vector, the largest eigenvalue of a square matrix, and an $N \times 1$ vector with all ones, respectively.

\section{Network And Channel Models}

Consider a cognitive network with a single primary user and a single cognitive (secondary) user as depicted in Fig. 1. Each user consists of a transmitter and a receiver. The primary transmitter and receiver are equipped with $N_{t}^{\mathrm{P}}$ and $N_{r}^{\mathrm{P}}$ antennas, respectively. Likewise, the secondary transmitter and receiver are equipped with $N_{t}^{\mathrm{C}}$ and $N_{r}^{\mathrm{C}}$ antennas, respectively. All antennas are assumed to be uncorrelated. Furthermore, we assume that the channel is frequency non-selective which can be easily achieved by using multiple-input multipleoutput orthogonal frequency division multiplexing (MIMOOFDM) [14]. Note that, however, our solution is not directly related to the channel model. Once channel information is known, the cognitive transmitter and receiver can compute the transmit/recive beamforming vectors using the proposed algorithms.

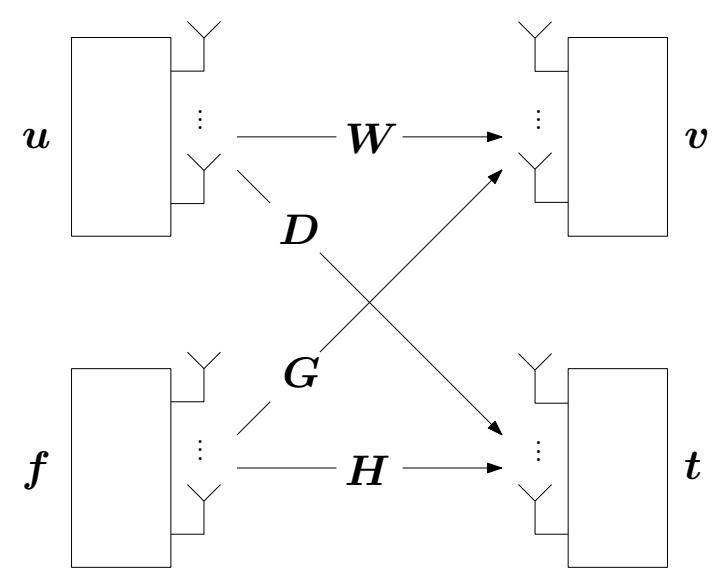

Fig. 1. The network model under consideration. The primary user is equipped with $N_{t}^{\mathrm{P}}$ and $N_{r}^{\mathrm{P}}$ antennas at the transmitter and receiver, respectively. The cognitive user is equipped with $N_{t}^{\mathrm{C}}$ and $N_{r}^{\mathrm{C}}$ antennas at the transmitter and receiver, respectively.

The MIMO channel between the primary transmitter and receiver is denoted by $\boldsymbol{W}$ whereas the one between the secondary transmitter and receiver is denoted by $\boldsymbol{H}$. The interference channel from the primary transmitter to the secondary receiver is denoted by $D$ and the interference channel from the secondary transmitter to the primary receiver is denoted by $G$. We model the individual channel elements in $\boldsymbol{W}, \boldsymbol{H}, \boldsymbol{D}$, and $\boldsymbol{G}$, as independent and identically distributed (i.i.d.) zeromean complex Gaussian random variables with unit variance

\footnotetext{
${ }^{1}$ We still use bold upper case letters for vector channels.
}

(Rayleigh fading). The primary transmitter employs a beamforming vector $\boldsymbol{u}$ for the transmission of its data symbol $x_{\mathrm{P}}$. Note that we consider the transmission of a single stream of information in the primary link. Of course this is not optimal from the information theory perspective. However, we note that this assumption is not restrictive. The results presented in this paper is also valid for the case of spatial multiplexing. ${ }^{2}$ We consider single stream of information only for ease of conveying the main idea. At the cognitive link, the transmitter employs a beamforming vector $\boldsymbol{f}$ for the transmission of its data symbol $x_{\mathrm{C}} . x_{\mathrm{P}}$ and $x_{\mathrm{C}}$ are assumed to be complex zero-mean unit variance random variables. Furthermore, let $v$ and $t$ be the receive combining vector for the primary and secondary receiver, respectively. Finally, we impose a unit energy constraint on all beamforming vectors, i.e., $\boldsymbol{u}^{*} \boldsymbol{u}=$ $\boldsymbol{f}^{*} \boldsymbol{f}=\boldsymbol{v}^{*} \boldsymbol{v}=\boldsymbol{t}^{*} \boldsymbol{t}=1$.

Let $P_{\mathrm{P}}$ and $P_{\mathrm{C}}$ be the transmit power at the primary and secondary transmitter, respectively, the received signal at the primary receiver and the secondary receiver are given respectively by

$$
r_{\mathrm{P}}=\sqrt{P_{\mathrm{P}}} \boldsymbol{v}^{*} \boldsymbol{W} \boldsymbol{u} x_{\mathrm{P}}+\sqrt{P_{\mathrm{P}}} \boldsymbol{v}^{*} \boldsymbol{G} \boldsymbol{f} x_{\mathrm{C}}+\boldsymbol{v}^{*} \boldsymbol{n}_{\mathrm{P}}
$$

and

$$
r_{\mathrm{C}}=\sqrt{P_{\mathrm{C}}} \boldsymbol{t}^{*} \boldsymbol{H} \boldsymbol{f} x_{\mathrm{C}}+\sqrt{P_{\mathrm{C}}} \boldsymbol{t}^{*} \boldsymbol{D} \boldsymbol{u} x_{\mathrm{P}}+\boldsymbol{t}^{*} \boldsymbol{n}_{\mathrm{C}} .
$$

The elements in the noise vectors $\boldsymbol{n}_{\mathrm{P}}$ and $\boldsymbol{n}_{\mathrm{C}}$ are modeled as i.i.d. zero-mean complex Gaussian random variables with variance $\sigma_{\mathrm{P}}^{2}$ and $\sigma_{\mathrm{C}}^{2}$, respectively. The resulting signal-tointerference-plus-noise ratio (SINR) of the primary and cognitive links are given by

$$
\mathrm{SINR}_{\mathrm{P}}=\frac{P_{\mathrm{P}} \boldsymbol{v}^{*} \boldsymbol{W}^{*} \boldsymbol{u} \boldsymbol{u}^{*} \boldsymbol{W} \boldsymbol{v}}{P_{\mathrm{P}} \boldsymbol{v}^{*} \boldsymbol{G} \boldsymbol{f} \boldsymbol{f}^{*} \boldsymbol{G}^{*} \boldsymbol{v}+\boldsymbol{v}^{*} \boldsymbol{v} \sigma_{\mathrm{P}}^{2}}
$$

and

$$
\mathrm{SINR}_{\mathrm{C}}=\frac{P_{\mathrm{C}} \boldsymbol{t}^{*} \boldsymbol{H}^{*} \boldsymbol{f} \boldsymbol{f}^{*} \boldsymbol{H} \boldsymbol{t}}{P_{\mathrm{C}} \boldsymbol{t}^{*} \boldsymbol{D} \boldsymbol{u} \boldsymbol{u}^{*} \boldsymbol{D}^{*} \boldsymbol{t}+\boldsymbol{t}^{*} \boldsymbol{t} \sigma_{\mathrm{C}}^{2}},
$$

respectively. It is obvious from (3) and (4) that in order to achieve zero interference, the beamforming vectors $v, f, t$, and $\boldsymbol{u}$ have to be designed such that $\boldsymbol{v}^{*} \boldsymbol{G} \boldsymbol{f}=0$ and $\boldsymbol{t}^{*} \boldsymbol{D} \boldsymbol{u}=$ 0 . In addition to gurantee zero interference, our goal is also to maximize the sum rate. For a single stream transmission, the sum rate is given by [15]

$$
\mathrm{R}_{\mathrm{S}}=\log _{2}\left(1+\mathrm{SINR}_{\mathrm{P}}\right)+\log _{2}\left(1+\operatorname{SINR}_{\mathrm{C}}\right) .
$$

Therefore, the design problem can be mathematically formulated as given at the top of this page.

In the next section, we present three solutions to the above optimization problem assuming that the primary user has completely no knowledge of the secondary user while achieving zero interference at both receivers.

\section{BEAMForming VeCtor Designs}

Considering the first constraint of (6), it is obvious that zero interference can be achieved by appropriately designing $v$ or $\boldsymbol{f}$ and $\boldsymbol{t}$ or $\boldsymbol{u}$. We assume that the cognitive user is transparent to the primary user since the performance of the primary

\footnotetext{
${ }^{2}$ In this case, the beamforming vectors become precoding matrices
} 


$$
\begin{aligned}
&\left\{\boldsymbol{v}_{\mathrm{opt}}, \boldsymbol{f}_{\mathrm{opt}}, \boldsymbol{t}_{\mathrm{opt}}, \boldsymbol{u}_{\mathrm{opt}}\right\}=\begin{array}{l}
\operatorname{argmax}\left\{\log _{2}\left(1+\mathrm{SINR}_{\mathrm{P}}\right)+\log _{2}\left(1+\mathrm{SINR}_{\mathrm{C}}\right)\right\} \\
\boldsymbol{v}, \boldsymbol{f}, \boldsymbol{t}, \boldsymbol{u}
\end{array}\left\{\begin{array}{l}
\boldsymbol{v}^{*} \boldsymbol{G} \boldsymbol{f}=0 \text { and } \boldsymbol{t}^{*} \boldsymbol{D} \boldsymbol{u}=0 \\
\boldsymbol{u}^{*} \boldsymbol{u}=\boldsymbol{f}^{*} \boldsymbol{f}=\boldsymbol{v}^{*} \boldsymbol{v}=\boldsymbol{t}^{*} \boldsymbol{t}=1
\end{array}\right. \\
& \text { subject to } \\
&\left\{\boldsymbol{v}_{\mathrm{opt}}, \boldsymbol{f}_{\mathrm{opt}}, \boldsymbol{t}_{\mathrm{opt}}, \boldsymbol{u}_{\mathrm{opt}}\right\}=\quad \begin{array}{l}
\operatorname{argmax}\left\{\log _{2}\left(1+\mathrm{SINR}_{\mathrm{P}}\right)+\log _{2}\left(1+\mathrm{SINR}_{\mathrm{C}}\right)\right\} \\
\text { subject to } \boldsymbol{f}, \boldsymbol{u}
\end{array}\left\{\begin{array}{l}
\boldsymbol{f} \in \operatorname{Null}\left(\boldsymbol{v}^{*} \boldsymbol{G}\right) \text { and } \boldsymbol{t} \in \operatorname{Null}(\boldsymbol{D} \boldsymbol{u}) \\
\boldsymbol{u}^{*} \boldsymbol{u}=\boldsymbol{f}^{*} \boldsymbol{f}=\boldsymbol{v}^{*} \boldsymbol{v}=\boldsymbol{t}^{*} \boldsymbol{t}=1
\end{array}\right.
\end{aligned}
$$

user should not be affected by the secondary link. To achieve zero interference caused to the primary receiver, the secondary transmitter can beamform in the null space of $\boldsymbol{v}^{*} \boldsymbol{G}$. Likewise, at the cognitive receiver the receive beamforming vector $t$ can be designed such that it is in the null space of $\boldsymbol{D} \boldsymbol{u}$ in order to avoid the interference caused by the primary transmitter. Note that $\boldsymbol{v}^{*} \boldsymbol{G}$ is a $1 \times N_{t}^{\mathrm{C}}$ vector and the dimension of its null space is $N_{t}^{\mathrm{C}}-1$. Similarly, the dimension of $\boldsymbol{D} \boldsymbol{u}$ is $N_{r}^{\mathrm{C}} \times 1$ and the dimension of its null space is $N_{r}^{\mathrm{C}}-1$. The optimization problem in (6) now becomes (7) given at the top of this page.

Having achieved zero interference both at the primary and secondary receivers, the remaining question is how to maximize the sum rate in (5). The first constraint in (7) obviously suggests that $\boldsymbol{f}$ depends on $\boldsymbol{v}$ and $\boldsymbol{t}$ depends on $\boldsymbol{u}$. The sum rate optimal solution requires finding $\boldsymbol{v}$ and $\boldsymbol{u}$ such that $R_{\mathrm{S}}$ is maximized and the optimal solution requires the knowledge of $\boldsymbol{W}, \boldsymbol{H}, \boldsymbol{D}$, and $\boldsymbol{G}$, i.e., the global channel state information (CSI). This is not reasonable from the primary user's point of view because the primary user should not be required to know the existence of the secondary user. Therefore, it is reasonable for the primary user to simply optimize $\boldsymbol{v}$ and $\boldsymbol{u}$ to maximize its own rate assuming no interference from the secondary transmitter. After obtaining $\boldsymbol{v}$ and $\boldsymbol{u}$, the secondary user can choose $\boldsymbol{f}$ and $\boldsymbol{t}$ (which are functions of $\boldsymbol{v}$ and $\boldsymbol{u}$, respectively, cf. the first constraint of (7)) to maximize its own rate.

The rate of the primary user can be maximized by appropriately designing $\boldsymbol{v}$ and $\boldsymbol{u}$. Since no interference is created at the primary user and the only constraint for the beamforming vectors $\boldsymbol{v}$ and $\boldsymbol{u}$ is the energy constraint, standard approaches in existing literature can be used to design $\boldsymbol{v}$ and $\boldsymbol{u}$ to maximize the rate of the $N_{t}^{\mathrm{P}} \times N_{r}^{\mathrm{P}}$ interference-free MIMO link [16], [17]. Since we restrict ourselves to the transmission of a single stream of information, the spectral efficiency can be maximized by maximizing the SINR due to the monotonic property of the logarithm function. It is well known that the SINR maximizing receive beamformer for a point-topoint link is the maximal ratio combining beamformer. In this case, the receive beamforming vector is simply $\boldsymbol{v}_{\text {opt }}=$ $\boldsymbol{W} \boldsymbol{u} / \sqrt{\boldsymbol{u}^{*} \boldsymbol{W}^{*} \boldsymbol{W} \boldsymbol{u}}$. With this design and the zero interference condition, (1) becomes

$$
r_{\mathrm{P}}=\frac{\sqrt{P_{\mathrm{P}}} \boldsymbol{u}^{*} \boldsymbol{W}^{*} \boldsymbol{W} \boldsymbol{u}}{\sqrt{\boldsymbol{u}^{*} \boldsymbol{W}^{8} \boldsymbol{W} \boldsymbol{u}}} x_{\mathrm{P}}+\frac{\boldsymbol{u}^{*} \boldsymbol{W}^{*}}{\sqrt{\boldsymbol{u}^{*} \boldsymbol{W}^{*} \boldsymbol{W} \boldsymbol{u}}} \boldsymbol{n}_{\mathrm{P}}
$$

and the corresponding instantaneous SINR is given by

$$
\mathrm{SINR}_{\mathrm{P}}=\frac{P_{\mathrm{P}} \boldsymbol{u}^{*} \boldsymbol{W}^{*} \boldsymbol{W} \boldsymbol{u}}{\sigma_{P}^{2}} .
$$

Obviously, the spectral efficiency of the primary link $\log _{2}(1+$ SINR $_{\mathrm{P}}$ ) can be maximized by beamforming in the direction of the eigenvector corresponding to the largest eigenvalue of $\boldsymbol{W}^{*} \boldsymbol{W}$. We denote the optimal transmit beamforming vector as $\boldsymbol{u}_{\text {opt }}$.

Using again the monotonic property of the logarithm function, the spectral efficiency of the cognitive link can be maximized by maximizing SINR $_{C}$. To maximize the SINR of the cognitive communication link, the design of $\boldsymbol{f}$ and $\boldsymbol{t}$ is not as flexible as the one for $\boldsymbol{v}$ and $\boldsymbol{u}$. This is because the feasible value of $f$ and $t$ is now constrained by the zero interference requirement. Specifically, the optimal beamformers are given by

$$
\begin{aligned}
\left\{\boldsymbol{f}_{\mathrm{opt}}, \boldsymbol{t}_{\mathrm{opt}}\right\}=\underset{\boldsymbol{f}, \boldsymbol{t}}{\operatorname{argmax}}\left\{\frac{P_{\mathrm{C}} \boldsymbol{t}^{*} \boldsymbol{H} \boldsymbol{f} \boldsymbol{f}^{*} \boldsymbol{H}^{*} \boldsymbol{t}}{\boldsymbol{t}^{*} \boldsymbol{t} \sigma_{C}^{2}}\right\} \\
\text { subject to } \quad\left\{\begin{array}{l}
\boldsymbol{f} \in \operatorname{Null}\left(\boldsymbol{v}_{\mathrm{opt}}^{*} \boldsymbol{G}\right) \\
\boldsymbol{t} \in \operatorname{Null}\left(\boldsymbol{D} \boldsymbol{u}_{\mathrm{opt}}\right) . \\
\boldsymbol{f}^{*} \boldsymbol{f}=\boldsymbol{t}^{*} \boldsymbol{t}=1
\end{array}\right.
\end{aligned}
$$

We present three proposals for the design of $f$ and $t$ in the next three subsections.

\section{A. Method 1: Discrete Search}

Let $\mathcal{F}$ and $\mathcal{T}$ be the set of basis vectors which spans the null space of $\boldsymbol{v}_{\mathrm{opt}}^{*} \boldsymbol{G}$ and $\boldsymbol{D} \boldsymbol{u}_{\mathrm{opt}}$, respectively. Note that the cardinality of $\mathcal{F}$ and $\mathcal{T}$ are $N_{t}^{\mathrm{C}}-1$ and $N_{r}^{\mathrm{C}}-1$, respectively. The instantaneous SINR of the cognitive link given by

$$
\mathrm{SINR}_{\mathrm{C}}=\frac{P_{\mathrm{C}} \boldsymbol{t}^{*} \boldsymbol{H} \boldsymbol{f} \boldsymbol{f}^{*} \boldsymbol{H}^{*} \boldsymbol{t}}{\boldsymbol{t}^{*} \boldsymbol{t} \sigma_{C}^{2}}
$$

can be maximized by performing an exhaustive search in $\mathcal{F}$ and $\mathcal{T}$, i.e.,

$$
\left\{\boldsymbol{f}_{\text {discrete }}, \boldsymbol{t}_{\text {discrete }}\right\}=\underset{\boldsymbol{f} \in \mathcal{F}, \boldsymbol{t} \in \mathcal{T}}{\operatorname{argmax}}\left\{\frac{P_{\mathrm{C}} \boldsymbol{t}^{*} \boldsymbol{H} \boldsymbol{f} \boldsymbol{f}^{*} \boldsymbol{H}^{*} \boldsymbol{t}}{\boldsymbol{t}^{*} \boldsymbol{t} \sigma_{C}^{2}}\right\} .
$$

Note that for $N_{t}^{\mathrm{C}}=N_{r}^{\mathrm{C}}=2$, there is only one vector in the set $\mathcal{F}$ and $\mathcal{T}$. In general, $\left(N_{t}^{\mathrm{C}}-1\right) \times\left(N_{r}^{\mathrm{C}}-1\right)$ computations are required to obtain the best beamformers $\boldsymbol{f}_{\text {discrete }}$ and $\boldsymbol{t}_{\text {discrete }}$. Although zero interference can always be guaranteed at both 
receivers by selecting the beamformer pairs $f, t$ as in (13), the obtained solution is not optimal in the sense of maximum sum rate because the search in (13) is not carried out over the entire null space of $\boldsymbol{v}_{\mathrm{opt}}^{*} \boldsymbol{G}$ and $\boldsymbol{D} \boldsymbol{u}_{\mathrm{opt}}$.

\section{B. Method 2: Gradient Algorithm}

Since any vector in the null space of $\boldsymbol{v}_{\mathrm{opt}}^{*} \boldsymbol{G}$ and $\boldsymbol{D} \boldsymbol{u}_{\mathrm{opt}}$ satisfies the zero interference condition, there could be potentially other vectors in those spaces which yield a higher SINR $_{\mathrm{C}}$ than $\boldsymbol{f}_{\text {discrete }}$ and $\boldsymbol{t}_{\text {discrete }}$. Suppose the columns of $\widehat{G}$ and $\widehat{D}$ contain the basis vectors of the null space of $\boldsymbol{v}_{\mathrm{opt}}^{*} \boldsymbol{G}$ and $\boldsymbol{D} \boldsymbol{u}_{\mathrm{opt}}$, respectively. The optimal beamformers are in the form of

$$
f=\frac{\widehat{G} a}{\sqrt{a^{*} a}} \quad \text { and } \quad t=\frac{\widehat{D} b}{\sqrt{b^{*} b}},
$$

where $\boldsymbol{a} \in \mathbb{C}^{\left(N_{t}^{\mathrm{C}}-1\right) \times 1}$ and $\boldsymbol{b} \in \mathbb{C}^{\left(N_{r}^{\mathrm{C}}-1\right) \times 1}$. The constrained optimization problem in (10) can now be formulated as an unconstrained one whose goal is to find $\boldsymbol{a} \in \mathbb{C}^{\left(N_{t}^{\mathrm{C}}-1\right) \times 1}$ and $\boldsymbol{b} \in \mathbb{C}^{\left(N_{r}^{\mathrm{C}}-1\right) \times 1}$ such that the objective function in (10) is maximized, i.e.,

$$
\left\{\boldsymbol{a}_{\mathrm{opt}}, \boldsymbol{b}_{\mathrm{opt}}\right\}=\underset{\boldsymbol{a}, \boldsymbol{b}}{\operatorname{argmax}}\left\{\frac{P_{\mathrm{C}} \boldsymbol{b}^{*} \widehat{\boldsymbol{D}}^{*} \boldsymbol{H} \widehat{\boldsymbol{G}} \boldsymbol{a} \boldsymbol{a}^{*} \widehat{\boldsymbol{G}}^{*} \boldsymbol{H}^{*} \widehat{\boldsymbol{D}} \boldsymbol{b}}{\boldsymbol{b}^{*} \boldsymbol{b} \boldsymbol{a}^{*} \boldsymbol{a} \sigma_{C}^{2}}\right\} .
$$

Unfortunately, there is no closed-form solution to (15) and we have to resort to numerical methods to solve the problem. In particular, a simple gradient (steepest accent) algorithm can be used to obtain $\boldsymbol{a}_{\text {opt }}$ and $\boldsymbol{b}_{\text {opt }}$ and we denote the resulting solutions as $\boldsymbol{a}_{\mathrm{grad}}$ and $\boldsymbol{b}_{\mathrm{grad}}$. Suppose $f(\boldsymbol{a}[i], \boldsymbol{b}[i])$ is the objection function in (15), the gradient algorithm is given by

$$
\left[\begin{array}{l}
\boldsymbol{a}[i+1] \\
\boldsymbol{b}[i+1]
\end{array}\right]=\left[\begin{array}{l}
\boldsymbol{a}[i] \\
\boldsymbol{b}[i]
\end{array}\right]+\mu\left[\begin{array}{l}
\partial f(\boldsymbol{a}[i], \boldsymbol{b}[i]) / \partial \boldsymbol{a}[i]^{*} \\
\partial f(\boldsymbol{a}[i], \boldsymbol{b}[i]) / \partial \boldsymbol{b}[i]^{*}
\end{array}\right],
$$

where $i$ is the discrete iteration index and $\mu$ is the adaptation step size. Furthermore, the two gradients in (16) are given by (17) and (18) shown at the top of next page, where $K$ is an irrelevant constant. The time index $i$ is dropped in the two equations above for ease of presentation. In the next section, some guidelines in choosing the adaptation constant $\mu$ and the initial values $\boldsymbol{a}[1]$ and $\boldsymbol{b}[1]$ are provided.

\section{Method 3: Optimal Solution for $N_{r}^{\mathrm{C}}=2$}

As mentioned in the last section, there is no closed-form solution to (15). However, if we fix the number of receive antennas of the cognitive user to two, the joint optimization in (15) becomes a single (vector) variable optimization problem and a closed-form solution is feasible. We first rewrite (10) as

$$
\left\{\boldsymbol{f}_{\mathrm{opt}}, \boldsymbol{t}_{\mathrm{opt}}\right\}=\underset{\boldsymbol{f}, \boldsymbol{t}}{\operatorname{argmax}}\left\{\frac{P_{\mathrm{C}} \boldsymbol{f}^{*} \boldsymbol{H}^{*} \boldsymbol{t} \boldsymbol{t}^{*} \boldsymbol{H} \boldsymbol{f}}{\boldsymbol{t}^{*} \boldsymbol{t} \sigma_{C}^{2}}\right\},
$$

assuming the same constraints as in (11). Suppose $N_{r}^{\mathrm{C}}=2$, the null space of $\boldsymbol{D} \boldsymbol{u}_{\mathrm{opt}}$ is one dimensional. Assume that the null space of $\boldsymbol{D} \boldsymbol{u}_{\mathrm{opt}}$ is spanned by $\boldsymbol{t}_{0}$ and therefore, the receive beamforming vector at the cognitive receiver is given by $\boldsymbol{t}_{\mathrm{opt}}=\boldsymbol{t}_{0}$. Recall that the optimal beamformers are in the form of (14) and let

$$
\overline{\boldsymbol{h}}=\widehat{\boldsymbol{G}}^{*} \boldsymbol{H}^{*} \boldsymbol{t}_{0}
$$

the optimization problem in (19) becomes

$$
\left\{\boldsymbol{a}_{\mathrm{opt}}\right\}=\underset{\boldsymbol{a}}{\operatorname{argmax}}\left\{\frac{P_{\mathrm{C}} \boldsymbol{a}^{*} \overline{\boldsymbol{h}} \overline{\boldsymbol{h}}^{*} \boldsymbol{a}}{\boldsymbol{a}^{*} \boldsymbol{a} \sigma_{C}^{2}}\right\} .
$$

The argument in (21) (which is essentially the SINR of the cognitive user) is known as the generalized Rayleigh quotient and by invoking the Rayleigh's principle [18], it can be bounded by

$$
\frac{P_{\mathrm{C}} \lambda_{\min }\left(\overline{\boldsymbol{h}} \overline{\boldsymbol{h}}^{*}\right)}{\sigma_{\mathrm{C}}^{2}} \leq \operatorname{SINR}_{\mathrm{C}}=\frac{P_{\mathrm{C}} \boldsymbol{a}^{*} \overline{\boldsymbol{h}} \overline{\boldsymbol{h}}^{*} \boldsymbol{a}}{\boldsymbol{a}^{*} \boldsymbol{a} \sigma_{C}^{2}} \leq \frac{P_{\mathrm{C}} \lambda_{\max }\left(\overline{\boldsymbol{h}} \overline{\boldsymbol{h}}^{*}\right)}{\sigma_{\mathrm{C}}^{2}} .
$$

Therefore, SINR $\mathrm{C}_{\mathrm{C}}$ can be maximized by choosing $\boldsymbol{a}_{\mathrm{opt}}$ to be the eigenvector corresponding to the maximum eigenvalues of $\overline{\boldsymbol{h}} \overline{\boldsymbol{h}}^{*}$. Consequently, $\boldsymbol{f}_{\mathrm{opt}}=\widehat{\boldsymbol{G}} \boldsymbol{a}_{\mathrm{opt}}$. It is interesting to note that although there is no constraint on $\boldsymbol{a} \in \mathbb{C}^{\left(N_{t}^{\mathrm{C}}-1\right) \times 1}$, the optimal solution $\boldsymbol{a}_{\text {opt }}$ always lies on the unit ball.

\section{Numerical AND Simulation Results}

The sum rate $R_{S}$ in (5) as a function of the average $\mathrm{SNR} \triangleq$ $\mathbb{E}\left\{\mathrm{SINR}_{\mathrm{P}}\right\}=\mathbb{E}\left\{\mathrm{SINR}_{\mathrm{C}}\right\}$ for $N_{t}^{\mathrm{C}}=3$ and $N_{r}^{\mathrm{C}}=2$ is shown in Fig. 2. Note that since $N_{t}^{\mathrm{P}}=N_{r}^{\mathrm{P}}=1, \boldsymbol{W}$ is simply a scalar and $\boldsymbol{u}=\boldsymbol{v}=1$. The three methods presented in Section III are used to generate the beamformers. For comparison, the sum rate results of a network with a $1 \times 1$ primary link and a $1 \times 1,3 \times 2$, and $2 \times 1$ secondary link system assuming that the two links do not interfere with each others are also shown. In these scenarios, the beamformers $f$ and $t$ are designed only to maximize the rate of the secondary link assuming single stream without any interference nullification constraint. This essentially gives us an idea of the price has to be paid for interference nullification.

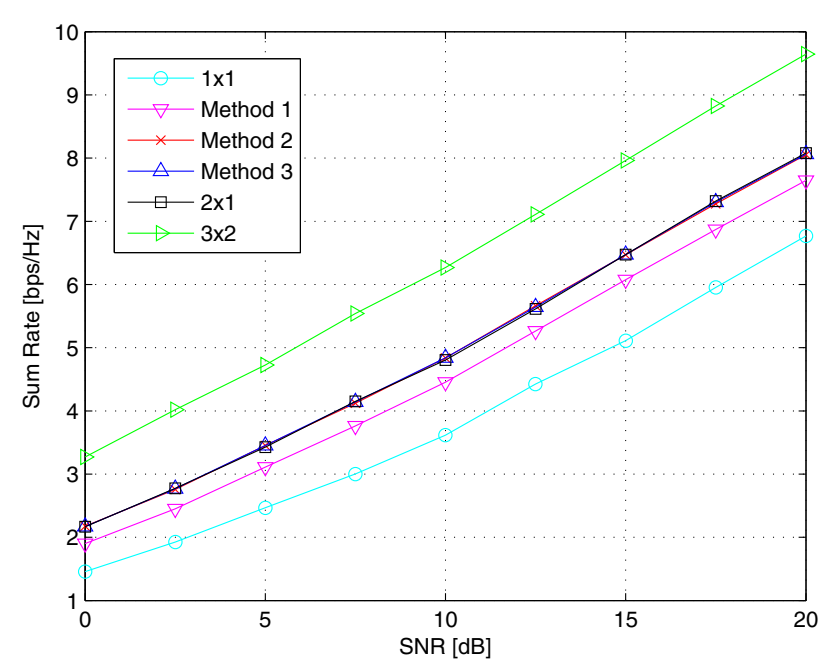

Fig. 2. Sum rate comparisons vs. SNR for a network with $N_{t}^{\mathrm{C}}=3$ 


$$
\begin{aligned}
& \frac{\partial f(\boldsymbol{a}[i], \boldsymbol{b}[i])}{\partial \boldsymbol{a}[i]^{*}}=K\left[\left(\left(\boldsymbol{b}^{*} \boldsymbol{b} \boldsymbol{a}^{*} \boldsymbol{a}\right)\right)\left(\widehat{\boldsymbol{G}}^{*} \boldsymbol{H}^{*} \widehat{\boldsymbol{D}} \boldsymbol{b} \boldsymbol{b}^{*} \widehat{\boldsymbol{D}}^{*} \boldsymbol{H} \widehat{\boldsymbol{G}} \boldsymbol{a}\right)-\left(\boldsymbol{a}^{*} \widehat{\boldsymbol{G}}^{*} \boldsymbol{H}^{*} \widehat{\boldsymbol{D}} \boldsymbol{b} \boldsymbol{b}^{*} \widehat{\boldsymbol{D}}^{*} \boldsymbol{H} \widehat{\boldsymbol{G}} \boldsymbol{a}\right)\left(\boldsymbol{b}^{*} \boldsymbol{b} \boldsymbol{a}\right)\right] \\
& \frac{\partial f(\boldsymbol{a}[i], \boldsymbol{b}[i])}{\partial \boldsymbol{b}[i]]^{*}}=K\left[\left(\boldsymbol{b}^{*} \boldsymbol{b} \boldsymbol{a}^{*} \boldsymbol{a}\right)\left(\widehat{\boldsymbol{D}}^{*} \boldsymbol{H} \widehat{\boldsymbol{G}} \boldsymbol{a} \boldsymbol{a}^{*} \widehat{\boldsymbol{G}}^{*} \boldsymbol{H}^{*} \widehat{\boldsymbol{D}} \boldsymbol{b}\right)-\left(\boldsymbol{b}^{*} \widehat{\boldsymbol{D}}^{*} \boldsymbol{H} \widehat{\boldsymbol{G}} \boldsymbol{a} \boldsymbol{a}^{*} \widehat{\boldsymbol{G}}^{*} \boldsymbol{H}^{*} \widehat{\boldsymbol{D}} \boldsymbol{b}\right)\left(\boldsymbol{a}^{*} \boldsymbol{a} \boldsymbol{b}\right)\right]
\end{aligned}
$$

As expected, the sum rate performance of beamformers obtained from Method 2 and Method 3 are identical and are better than the performance of the ones obtained from Method 1. In general, Method 2 and Method 3 are $1.4 \mathrm{~dB}$ better than Method 1 . Finally, the gap between the $3 \times 2$ curve and Method 3 curve is about $4.5 \mathrm{~dB}$ and it essentially represents the price that has to be paid in order to avoid cross interference between the primary and secondary links.

In the last example shown in Fig. 3, we consider a system with $N_{t}^{\mathrm{C}}=10$ assuming the rest of the parameters unchanged as in Fig. 2. Similar observation as in Fig. 2 can be made. In this case, Method 2 is about $5 \mathrm{~dB}$ better than Method 1. Also, it is noted that the gap between the Method 3 and $10 \times 2$ curves reduces to about $2 \mathrm{~dB}$. This is due to the diminishing of return phenomenon observed generally in MIMO system as the number of antennas increases.

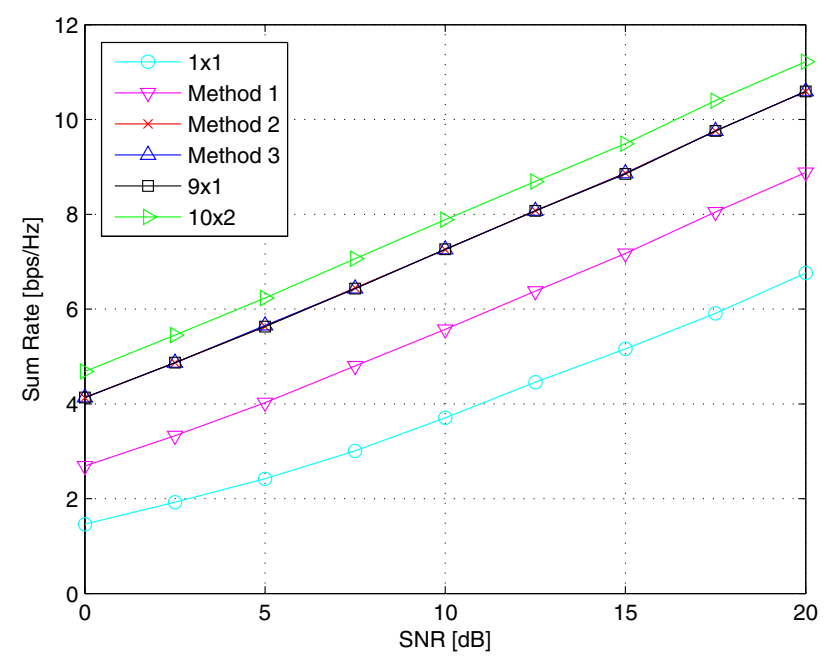

Fig. 3. Sum rate comparisons vs. SNR for a network with $N_{t}^{\mathrm{C}}=10$

\section{CONCLUSION}

In this paper, we considered interference cancellation and rate maximization via uncoordinated beamforming in a cognitive network which consists of a single primary and secondary user. The secondary cognitive user was allowed to transmit concurrently with the primary licensed user. The beamforming vectors of the cognitive user were designed such that the interference is completely nullified both at the primary and secondary receivers while maximizing the rate of the cognitive link. Since no interference is created at the primary receiver, traditional approaches can be used to design the beamforming vectors or precoding matrices of the primary user. Three approaches were proposed for the design of the beamforming vectors of the cognitive link. The optimal beamforming solution for the special case of $N_{r}^{\mathrm{C}}=2$ was also derived. For $N_{r}^{\mathrm{C}}>2$, we resorted to numerical methods to solve the optimization problem. Finally, it is noted that we motivate the uncoordinated beamforming and rate maximization concept in a cognitive network. However, the results can also be applied to practical systems, e.g., small cell deployment in a macro network.

\section{REFERENCES}

[1] United States frequency allocations: The radio spectrum. U.S. Department of Commerce, National Telecommunications and Information Administration, Office of Spectrum Management, October 2003.

[2] J. Mitola. Cognitive Radio. PhD thesis, Royal Institute of Technology (KTH), 2000.

[3] S. Srinivasa and S. A. Jafar. The throughput potential of cognitive radio: A theoretical perspective. IEEE Communications Magazine, 45(5):7379, May 2007.

[4] T. Yucek and H. Arslan. A survey of spectrum sensing algorithms for cognitive radio applications. IEEE ACommunications Surveys and Tutorials, 11(1):116-130, April 2009.

[5] Z. Quan, S. Cui, A. H. Sayed, and H. V. Poor. Optimal multiband joint detection for spectrum sensing in cognitive radio networks. IEEE Trans. Signal Processing, 57(3):1128-1140, March 2009.

[6] G. Ganesan and Y. Li. Cooperative spectrum sensing in cognitive radio networks. In Proceedings of IEEE Dynamic Spectrum Access Networks (DySPAN), pages 137-143, Baltimore, Maryland, USA, November 2005.

[7] S. M. Mishra, A. Sahai, and R. W. Brodersen. Cooperative sensing among cognitive radios. In Proceedings of IEEE International Conference on Communications (ICC), pages 1658-1663, Istanbul, Turkey, June 2006.

[8] R. Zhang and Y.-C. Liang. Exploiting multi-antennas for opportunistic spectrum sharing in cognitive radio networks. IEEE Jour. Sel. Top. in Sig. Proc., 2(1):88-102, 2008.

[9] B. D. V. Veen and K. M. Buckley. Beamforming: A versatile approach to spatial filtering. IEEE ASSP Magazine, pages 4-24, 1988.

[10] X. Jing and D. Raychaudhuri. A spectrum etiquette protocol for efficient coordination of radio devices in unlicensed bands. In Proceedings of IEEE Personal, Indoor and Mobile Radio Communications (PIMRC), pages 172-176, Beijing, China, September 2003.

[11] M. Buddhikot, P. Kolodzy, S. Miller, K. Ryan, and J. Evans. Dimsumnet: new directions in wireless networking using coordinated dynamic spectrum access. In Proceedings of IEEE International Symposium on a World of Wireless Mobile and Multimedia Networks (WoWMoM), pages 78-85, Taormina, Giardini Naxos, June 2005.

[12] J. Perez-Romero, O. Sallent, R. Agusti, and L. Giupponi. A novel ondemand cognitive pilot channel enabling dynamic spectrum allocation. In Proceedings of IEEE Dynamic Spectrum Access Networks (DySPAN), pages 46-54, Dublin, Ireland, April 2007.

[13] S. Panichpapiboon and J. M. Peha. Providing secondary access to licensed spectrum through coordination. Wireless Networks, 14(3):295307, June 2008 .

[14] H. Bolcskei. MIMO-OFDM wireless systems: basics, perspectives, and challenges. IEEE Wireless Communications Magazine, 13(4):31-37, August 2006

[15] J. G. Proakis. Digital Communications. McGraw-Hill, New York, forth edition, 2000.

[16] G. J. Foschini. Layered space-time architecture for wireless communication in a fading environment when using multi-element antennas. Bell Labs Technical Journal, 1(2):41-59, 1996.

[17] G. J. Foschini, G. D. Golden, R. A. Valenzuela, and P. W. Wolniansky. Simplified processing for high spectral efficiency wireless communication employing multi-element arrays. IEEE Jour. Sel. Areas in Commun., 17(11):1841-1852, November 1999.

[18] K. Washizu. On the bounds of eigenvalues. Quarterly Journal of Mechanics \& Applied Mathematics, 8(3):311-325, 1955. 